Case report

\title{
Panniculitis in a patient presenting with a pancreatic tumour and polyarthritis: a case report Carolyn Chee ${ }^{1}$
}

Address: Nottingham City Hospital, Nottingham University Hospitals NHS Trust City Campus, Hucknall Road, Nottingham NG5 1PB, UK Email: CC - carolyn_cwl@hotmail.com

Received: II November 2008 Accepted: 29 January 2009 Published: 6 July 2009

Journal of Medical Case Reports 2009, 3:733I doi: 10.4076/I752-|947-3-733।

This article is available from: http://jmedicalcasereports.com/jmedicalcasereports/article/view/733।

(c) 2009 Chee; licensee Cases Network Ltd.

This is an Open Access article distributed under the terms of the Creative Commons Attribution License (http://creativecommons.org/licenses/by/3.0), which permits unrestricted use, distribution, and reproduction in any medium, provided the original work is properly cited.

\begin{abstract}
Introduction: Panniculitis is a rare manifestation of pancreatic disease. Rarer still is the association of panniculitis, pancreatitic disease and polyarthritis. A literature search revealed less than five cases of pancreatic panniculitis associated with pancreatic tumour and polyarthritis.

Case presentation: An 84-year-old Caucasian man presented with epigastric pain, weight loss, polyarthritis and multiple discharging nodules. A computed tomography scan revealed a mass in the head of the pancreas. Histology of the cutaneous lesions confirmed the diagnosis of pancreatic panniculitis.

Conclusion: Pancreatic panniculitis can clinically present in many ways to clinicians across a broad scope of specialties. Knowledge and understanding of the association between panniculitis and polyarthritis with pancreatic disease may aid rapid diagnosis and management.
\end{abstract}

\section{Introduction}

Pancreatic panniculitis is a rare disease involving fat necrosis in the panniculus in association with pancreatic disease. The rarity of this condition is best described by Mullin et al. who reported one case of panniculitis out of 893 patients with various pancreatic diseases [1]. We report a man presenting with panniculitis, pancreatic tumour and polyarthritis with a fatal outcome. Awareness of the rare skin manifestation observed and the potentially fatal consequences, can guide physicians to rapid diagnosis and management.

\section{Case presentation}

An 84-year-old Caucasian man presented with a 4-week history of severe abdominal pain radiating to his back and with haematuria. He was receiving long-term warfarin treatment for atrial flutter and his international normalised ratio on admission was 17.6. He complained of nausea and weight loss, but reported no recent change in bowel habit.

On examination, the patient was cachectic and pyrexial. He had epigastric and right upper quadrant tenderness. Of note was swelling of his right carpometacarpal, bilateral thumb and index finger metacarpal joints.

Laboratory investigations revealed a white-cell count of $12.6 \times 10^{9} / \mathrm{L}$, C-reactive protein of $198 \mathrm{mg} / \mathrm{L}$, gammaglutamyl transpeptidase of 273, raised serum lipase of $17362 \mathrm{U} / \mathrm{L}$ and serum amylase of $619 \mathrm{U} / \mathrm{L}$. Chest and 


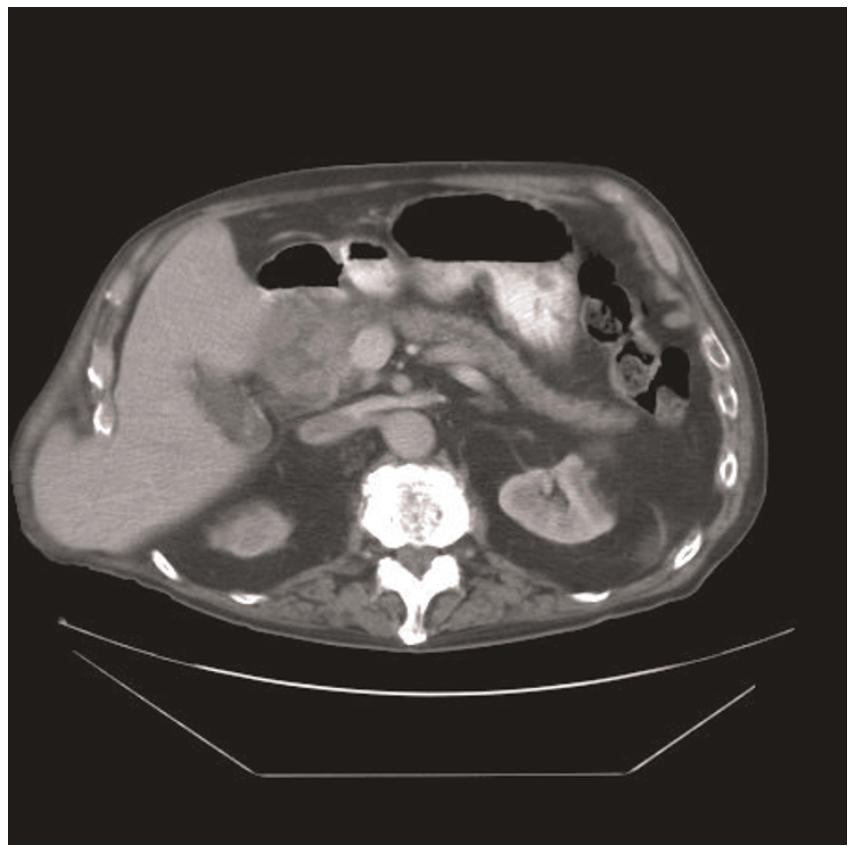

Figure I. Computed tomography scan of the abdomen showing a mixed attenuation mass of the head of pancreas.

abdominal X-rays were normal. The abdominal computed tomography (CT) scan showed a $9 \times 8 \mathrm{~cm}$ mixed attenuation mass initially thought to represent pancreatic head haematoma (Figure 1). The patient's warfarin treatment was discontinued and he was managed conservatively with intravenous fluids, analgesia and bed rest. Levels of the tumour markers carcinoembryonic antigen
(CEA) and CA19-9 were normal. A follow-up scan 8 weeks later revealed that the mass had not resolved and was now more likely to represent a tumour of the head of the pancreas or a pancreatic pseudocyst. The patient declined an endoscopic ultrasound.

He returned four weeks later, complaining of fever, left knee pain and a pus-like discharge from his hands and feet. Blood and knee-joint fluid cultures were negative. An $\mathrm{x}$-ray of his knees showed features of osteoarthritis. A whole-body bone scan excluded the possibility of bone metastases. A few days later, the patient developed multiple tender red, brownish nodules on his lower limbs and discharging lesions on his hands (Figures 2A and $\mathrm{B}$ ). He also had a discharging wound on his right buttock. He was referred to the Department of Dermatology where the nodules were thought to represent paraneoplastic lesions or a skin manifestation of atypical Mycobacterium infection. The first skin biopsy showed nonspecific inflammation of erythema nodosum.

A second skin biopsy revealed extensive areas of necrotic subcutaneous fat surrounded by florid acute and chronic inflammation consistent with pancreatic panniculitis (Figure 3 ). The patient declined investigations and treatment of the pancreatic mass. Management was primarily supportive and his condition deteriorated rapidly. He was eventually referred to the palliative care team for pain and symptom control. The patient died 5 months after his initial presentation to our hospital.

\section{Discussion}

Pancreatic panniculitis affects $2 \%$ to $3 \%$ of patients with pancreatic disease [2]. This involves fat necrosis in the
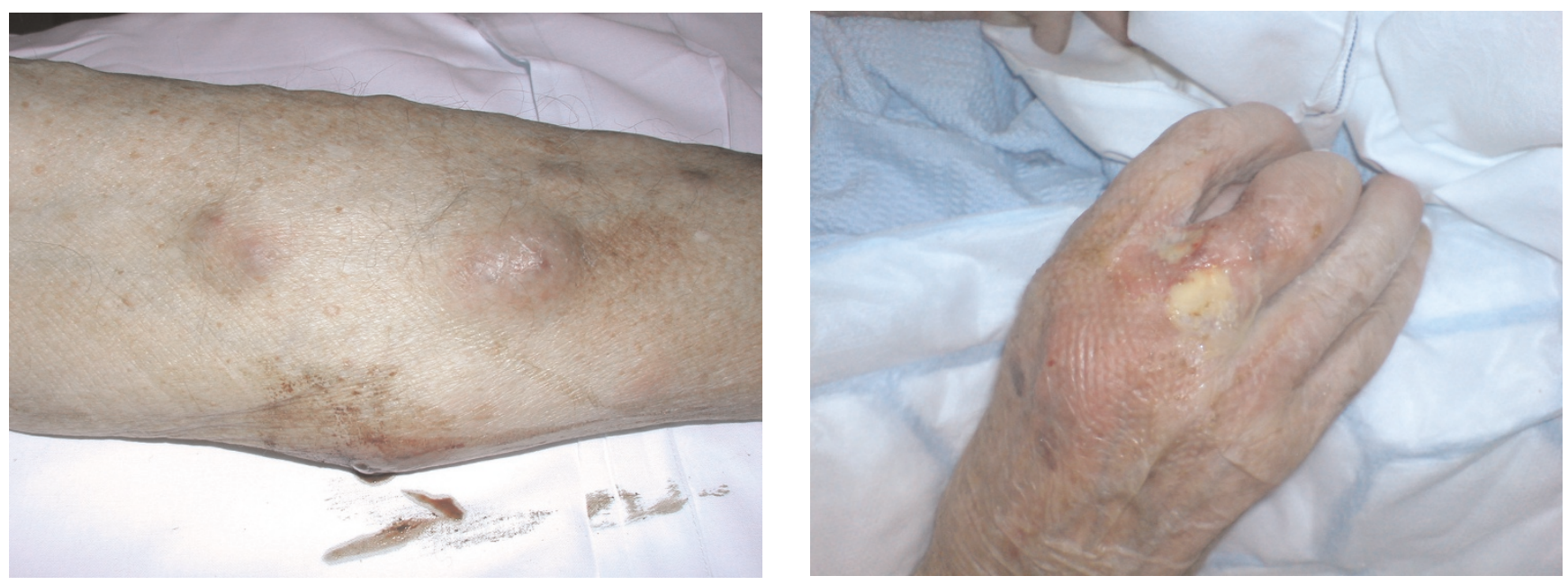

Figure 2. (A) Multiple reddish-purple nodules on lower limb and (B) discharging lesion on hand. 


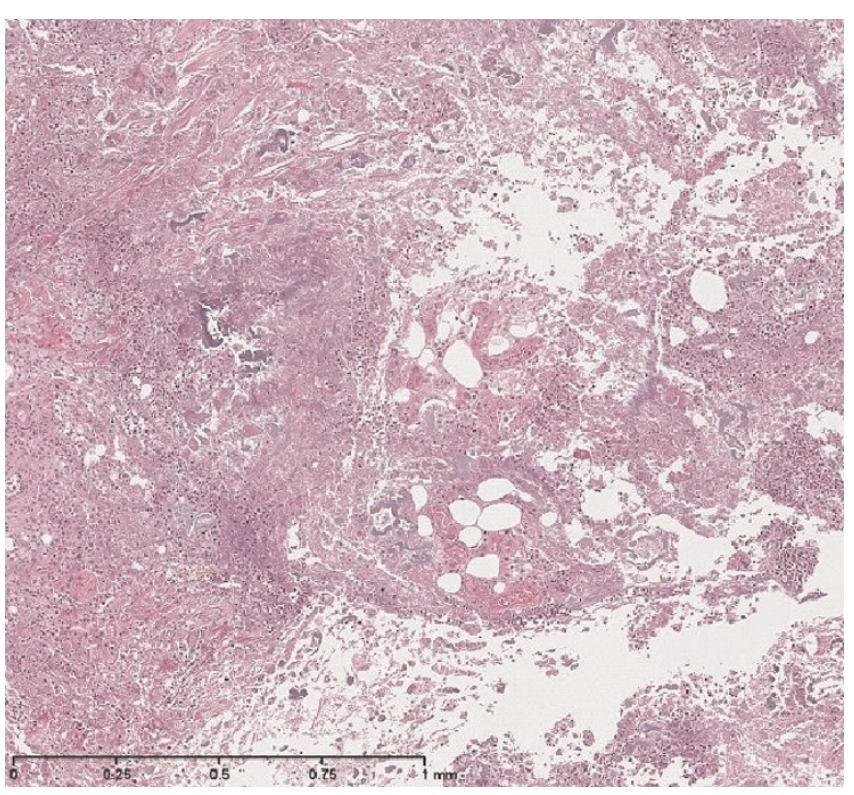

Figure 3. Histopathological sample showing areas of fat necrosis (haematoxylin stain, $\times 100$ ).

panniculus and distant foci in pancreatic diseases such as acute or chronic pancreatitis and pancreatic carcinoma. It is less commonly associated with pancreatic pseudocyst, post-traumatic pancreatitis, pancreatic divisum or haemophogocytic syndrome [3]. The pathogenesis is unknown but has been postulated to involve the release of pancreatic enzymes, particularly amylase and lipase from the diseased pancreas, causing subcutaneous fat necrosis [4]. Skin histopathology findings of panniculitis are virtually pathognomonic, described as ghost-like anucleated cells with shadowy walls [5]. Panniculitis manifests as tender erythematous nodules on the lower extremities but can also occur on the arms, trunk or back. These lesions may ulcerate and extrude an oily discharge [6]. Skin lesions are the presenting feature in about $40 \%$ of pancreatic panniculitis and in some cases precede development of pancreatic disease [2]. There may be joint manifestations of arthritis or arthralgia [7]. Not much is known of the underlying pathogenesis of arthropathy in pancreatic panniculitis. Some studies postulate that increased levels of lipolytic enzymes of pancreatic origin, once they freely circulate in the tissues, are cytotoxic for many cells including leukocytes and therefore perpetuate inflammation. Synovial fluid of patients with pancreatic arthritis syndrome has been shown to contain elevated concentrations of these lipolytic enzymes [8,9].

Several cases of a triad of panniculitis, pancreatitis and polyarthritis have been reported. Development of pancreatitis in these cases was related to high levels of alcohol intake [10-12]. Treatment is largely supportive but, where possible, should involve treatment of the underlying cause. Pancreatic panniculitis has a high mortality rate unless the underlying pancreatic abnormality is reversed [8]. Several case studies have shown resolution of panniculitis in patients after surgical intervention or medical management of their underlying pancreatic disease $[3,13]$. Prognosis is worse in cases of pancreatic panniculitis associated with pancreatic tumour [14].

In our patient, the clinical finding of panniculitis, pancreatic mass on CT scan, elevated pancreatic enzymes and histological findings of pancreatic panniculitis strengthened the likelihood of a pancreatic tumour in the absence of histopathological and post-mortem biopsies of the pancreatic mass.

\section{Conclusion}

As patients may present to practitioners of various medical specialities with differing symptoms or signs, awareness of the relationship between panniculitis lesions, pancreatic disease and arthritis or arthralgia may prevent a delay of prompt diagnosis and management.

\section{Consent}

As the patient died before the write-up of his case, written informed consent was obtained from the patient's nextof-kin for the publication of this case report and any accompanying images. A copy of the written consent is available for review by the Editor-in-Chief of this journal.

\section{Competing interests}

The author declares that she has no competing interests.

\section{Acknowledgements}

The author would like to acknowledge Dr A Khan of the Department of Pathology and Dr N Bassi of the Department of Dermatology, Nottingham City Hospital, who contributed towards the acquisition of histopathology and clinical images, respectively.

\section{References}

I. Mullin GT, Caperton GM Jr, Crespin SR, Williams RC Jr: Arthritis and skin lesions resembling erythema nodosum in pancreatic disease. Ann Intern Med 1968, 68:75-87.

2. Dahl PR, Su WP, Cullimore KC, Dicken $\mathrm{CH}$ : Pancreatic Panniculitis. J Am Acad Dermatol 1995, 33:4I3-4I7.

3. Johnson MA, Kannan DG, Balachandar TG, Jeswanth S, Rajendran S, Surendran R: Acute septal panniculitis. A cutaneous marker of a very early stage of pancreatic panniculitis indicating acute pancreatitis. JOP 2005, 6:334-338.

4. Berman B, Conteas C, Smith B, Leong S, Hornbeck L 3rd: Fatal pancreatitis presenting with subcutaneous fat necrosis. Evidence that lipase and amylase alone do not induce lipocyte necrosis. J Am Acad Dermatol 1987, I 7:359-364.

5. Lee WS, Kim MY, Kim SW, Paik CN, Kim HO, Park YM: Fatal pancreatic panniculitis associated with acute pancreatitis: a case report. J Korean Med Sci 2007, 22:914-917.

6. Romero G, Vanachocha F: Pancreatic panniculitis. Dermatol Clin 2008, 26:465-470. 
7. Zellman GL: Pancreatic panniculitis. J Am Acad Dermatol 1996, 35:282-283.

8. Wilson HA, Askari AD, Neiderhiser DH, Johnson AM, Andrews BS, Hoskins LC: Pancreatitis with arthropathy and subcutaneous fat necrosis. Arthritis Rheum 1983, 26:121-126.

9. Simkin P, Brunzell J, Wisner D, Fiechtner JJ, Carlin JS, Willkens RF: Free fatty acids in the pancreatitic arthritis syndrome. Arthritis Rheum 1983, 26:127-132.

10. Mourad FH, Hannoush HM, Bahlawan M, Uthman I, Uthman S: Panniculitis and arthritis as the presenting manifestation of chronic pancreatitis. J Clin Gastroenterol 200I, 32:259-26I.

II. Price-Forbes AN, Filer A, Udeshi UL, Rai A: Progression of imaging in pancreatitis panniculitis polyartritis (PPP) syndrome. Scand J Rheumatol 2006, 35:72-77.

12. Watts RA, Kelly S, Hacking JC: Fat Necrosis. An unusual cause of polyarthritis. J Rheumatol 1993, 20:1432-I435.

13. Outtas O, Barthat M, De Troger J, Franck F, Garcia S: Pancreatic panniculitis with intraductal carcinoid tumour of the pancreas divisum. Ann Dermatol Venereol 2004, I 3 I:466-469.

14. Van der Zee JA, van der Hillegersberg R, Toonstra J, Gouma DJ: Subcutaneous nodules pointing towards pancreatic disease: pancreatic panniculitis. Dig Surg 2004, 2 I:275-276.

\section{Do you have a case to share?}

Submit your case report today

- Rapid peer review

- Fast publication

- PubMed indexing

- Inclusion in Cases Database

\section{Any patient, any case, can teach us something}

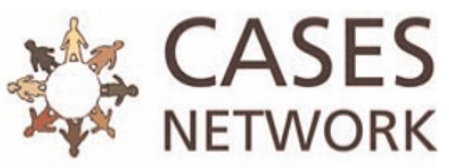

www.casesnetwork.com 